\title{
NOTES ON SOME OF KAHANE'S WORKS: INGHAM TYPE THEOREMS AND BERNOULLI CONVOLUTIONS
}

\author{
K. DAJANI ${ }^{1}$, V. KOMORNIK ${ }^{2, *, \dagger}$ and P. LORETI ${ }^{3}$ \\ ${ }^{1}$ Department of Mathematics, Utrecht University, Budapestlaan 6, P.O. Box 80.000, \\ 3508 TA Utrecht, The Netherlands \\ e-mail:k.dajani1@uu.nl \\ ${ }^{2}$ Département de mathématique, Université de Strasbourg, 7 rue René Descartes, \\ 67084 Strasbourg Cedex, France \\ e-mail: komornik@math.unistra.fr \\ ${ }^{3}$ Sapienza Università di Roma, Dipartimento di Scienze di Base e Applicate per l'Ingegneria, \\ via A. Scarpa n. 16, 00161 Roma, Italy \\ e-mail: paola.loreti@sbai.uniroma1.it
}

(Received March 21, 2018; revised May 5, 2018; accepted May 5, 2018)

Dedicated to the memory of Jean-Pierre Kahane

\begin{abstract}
We discuss the optimality of Kahane's multidimensional Ingham type theorem and we establish a relationship between the absolute continuity of Bernoulli convolutions and some results on expansions in non-integer bases.
\end{abstract}

\section{A multidimensional Ingham type theorem}

In this section we consider functions of the form

$$
x(t)=\sum_{k \in K} x_{k} e^{i \omega_{k} \cdot t}, \quad t \in \mathbb{R}^{N},
$$

with square summable complex coefficients $x_{k}$, where $\left(\omega_{k}\right)_{k \in K}$ is a given family of vectors in $\mathbb{R}^{N}$, and $\omega_{k} \cdot t$ denotes the usual scalar product in $\mathbb{R}^{N}$. For $N=1, K=\mathbb{Z}$ and $\omega_{k}=k$ they reduce to the classical trigonometric series.

\footnotetext{
* Corresponding author.

$\dagger$ The second author was partially sponsored by NWO Traveler's Grant no. 040.11.599/2886.

Key words and phrases: Bernoulli convolution, expansion in non-integer bases.

Mathematics Subject Classification: primary 11A63.
} 
We are interested in the following generalization of Parseval's theorem. Given an Euclidean ball $B_{R} \subset \mathbb{R}^{N}$ of radius $R$, we investigate the validity of the estimates

$$
c_{1} \sum_{k \in K}\left|x_{k}\right|^{2} \leq \int_{B_{R}}|x(t)|^{2} d t \leq c_{2} \sum_{k \in K}\left|x_{k}\right|^{2}
$$

with suitable (strictly) positive constants $c_{1}$ and $c_{2}$, independent of the particular choice of the coefficients $x_{k}$.

Such estimates cannot hold unless the family $\left(\omega_{k}\right)_{k \in K}$ satisfies the following uniform gap condition: there exists a positive number $\gamma$ such that

$$
\left\|\omega_{k}-\omega_{n}\right\| \geq \gamma \quad \text { for all } k \neq n .
$$

In the one-dimensional case Ingham proved that under this condition the estimates (1.2) hold whenever $R>\pi / \gamma$, i.e., for all intervals $B_{R}$ of length greater than $2 \pi / \gamma$. Adapting his proof to several dimensions, Kahane [12] proved the following:

THEOREM 1.1. If (1.3) is satisfied, then the estimates (1.2) hold for all balls of radius $R>c_{N} / \gamma$ where $c_{N}$ is a suitable explicit constant depending only on $N$.

Kahane's constants $c_{N}$ were improved by a different proof in [2], [3] and [17] as follows:

THEOREM 1.2. If (1.3) is satisfied, then the estimates (1.2) hold for all balls of radius $R>\sqrt{\mu}$, where $\mu$ denotes the first eigenvalue of $-\Delta$ in the Sobolev space $\mathcal{H}_{0}^{1}\left(B_{\gamma / 2}\right)$.

As usual, the Sobolev space $\mathcal{H}^{1}(\Omega)$, where $\Omega$ is a non-empty bounded domain of $\mathbb{R}^{N}$, is formed by those functions of the Lebesgue space $L^{2}(\Omega)$ whose first-order partial derivatives also belong to $L^{2}(\Omega)$, and $\mathcal{H}_{0}^{1}(\Omega)$ denotes its closed linear subspace spanned by the $C^{\infty}$ functions $f: \Omega \rightarrow \mathbb{R}$ vanishing outside some compact set $K_{f} \subset \Omega$.

In the one-dimensional case this reduces to Ingham's theorem because the first eigenfunction of $-\Delta$ in $\mathcal{H}_{0}^{1}(-\gamma / 2, \gamma / 2)$ is (up to a multiplicative constant) the function $\cos (\pi x / \gamma)$, so that $\mu=\pi^{2} / \gamma^{2}$.

In the above references more general results were obtained, by replacing the Euclidean norms in the gap condition by arbitrary $L^{p}$ norms with $1 \leq p \leq \infty$. Furthermore, the corresponding conditions $R>\sqrt{\mu_{p}}$, where $\mu_{p}$ denotes the first eigenvalue of $-\Delta$ in the Sobolev space $\mathcal{H}_{0}^{1}\left(B_{\gamma / 2}^{p}\right)$, were proved to be optimal if $p=\infty$, and also if $p=1$ and $N=2$. It is an interesting open question whether this condition is optimal for the other values of $p$. 
Let us recall the crucial part of the proof of Theorem 1.2: the left-hand side inequality of (1.2).

Let us denote by $H$ and $\mu$ the first eigenfunction and eigenvalue of $-\Delta$ in $\mathcal{H}_{0}^{1}\left(B_{\gamma / 2}\right)$. We may assume that $H>0$ in $B_{\gamma / 2}$. Extending by zero outside this ball, we obtain a continuous function on $\mathbb{R}^{N}$, still denoted by $H$. Setting $G:=\left(R^{2}+\Delta\right)(H * H)$ and introducing their Fourier transforms

$$
h(t):=\int_{B_{\gamma}} H(x) e^{-i x \cdot t} d x \text { and } g(t):=\int_{B_{\gamma}} G(x) e^{-i x \cdot t} d x,
$$

we have $G \in \mathcal{H}_{0}^{1}\left(B_{\gamma}\right)$ and

$$
g(t)=\left(R^{2}-|t|^{2}\right)|h(t)|^{2} \leq 0 \quad \text { if }|t| \geq R .
$$

Hence $g$ is bounded from above in $\mathbb{R}^{N}$ by some constant $\alpha$. Applying the identity

$$
\int_{\mathbb{R}^{N}} g(t)|x(t)|^{2} d t=(2 \pi)^{N} \sum_{k, n \in K} x_{k} \overline{x_{n}} G\left(\omega_{k}-\omega_{n}\right)=(2 \pi)^{N} G(0) \sum_{k \in K}\left|x_{k}\right|^{2}
$$

(where in the last step we use the gap condition $G\left(\omega_{k}-\omega_{n}\right)=0$ whenever $k \neq n)$, it follows that

$$
(2 \pi)^{N} G(0) \sum_{k \in K}\left|x_{k}\right|^{2}=\int_{\mathbb{R}^{N}} g(t)|x(t)|^{2} d t \leq \alpha \int_{B_{R}}|x(t)|^{2} d t .
$$

This yields the first inequality of (1.2) with $c_{1}=(2 \pi)^{N} G(0) / \alpha$, provided that $G(0)>0$.

This last inequality follows from our assumption $R>\sqrt{\mu}$ and from the variational characterization of the first eigenvalue $\mu$ of the operator $-\Delta$ in $\mathcal{H}_{0}^{1}\left(B_{\gamma / 2}\right)$ :

$$
G(0)=\int_{B_{\gamma / 2}} R^{2} H^{2}-|\nabla H|^{2} d x=\left(R^{2}-\mu\right) \int_{B_{\gamma / 2}} H^{2} d x>0 .
$$

\section{Bernoulli convolutions and expansions in non-integer bases}

Given $1<q \leq 2$, the Bernoulli convolution $\nu_{q}$ is defined as the weak-star limit of the measures

$$
\nu_{q, n}:=\frac{1}{2^{n}} \sum_{a} \delta_{a}, \quad n=0,1, \ldots,
$$


where $a$ runs over the numbers

$$
a=\sum_{i=1}^{n} \frac{a_{i}}{q^{i}}, \quad a_{1} \cdots a_{n} \in\{0,1\}^{n}
$$

and $\delta_{x}$ denotes the Dirac measure at the point $x$. For example, $\nu_{2}$ is the Lebesgue measure on $[0,1]$.

The convolution terminology is explained by an alternative definition, used, e.g., in [4]. If we introduce the purely discrete measures $\delta_{i}$ on $\mathbb{R}$ by the formula

$$
\delta_{i}(\{0\})=\delta_{i}\left(\left\{q^{-i}\right\}\right)=\frac{1}{2},
$$

then

$$
\nu_{q, n}=\delta_{1} * \cdots * \delta_{n}
$$

for all $n$, and

$$
\nu_{q}=\lim _{n \rightarrow \infty} \delta_{1} * \cdots * \delta_{n} .
$$

Jessen and Wintner [11] proved that for each $q, \nu_{q}$ is either absolutely continuous or singular. Erdôs [5] proved that $\nu_{q}$ is singular for all Pisot numbers $1<q<2$. In the other direction, he proved in [6] that there exists a number $0<\varepsilon<1$ such that $\nu_{q}$ is absolutely continuous for almost every $1<q<1+\varepsilon$. Solomyak [18] proved that in fact $\nu_{q}$ is absolutely continuous for almost every $1<q<2$. Analyzing the proof in [6], Kahane [13] proved that the Hausdorff dimension of the exceptional set of $q$ 's in $(1,1+\varepsilon)$ tends to zero as $\varepsilon \rightarrow 0$.

We still do not have an algebraic characterization of the values $q$ for which $\nu_{q}$ is absolutely continuous. A bold conjecture is that $\nu_{q}$ is absolutely continuous for all non-Pisot numbers $1<q<2$.

The purpose of this section is to relate this problem to some results on expansions in non-integer bases. For this we recall in Propositions 2.1-2.3 some recent results of Kempton [14].

We fix base $1<q<2$ and we introduce the interval $I_{q}:=\left[0, \frac{1}{q-1}\right]$. The first result is straightforward:

Proposition 2.1. The formula

$$
(P f)(x):=\frac{q}{2}(f(q x)+f(q x-1)), \quad x \in \mathbb{R}
$$

defines a positive linear operator $P: L^{1}(\mathbb{R}) \rightarrow L^{1}(\mathbb{R})$, satisfying the following two extra conditions:

- $\int P f d x=\int f d x$ for all $f \in L^{1}(\mathbb{R})$;

- if $f$ vanishes outside $I_{q}$, then $P f$ also vanishes outside $I_{q}$. 
The second result follows from the self-similarity of Bernoulli convolutions:

Proposition 2.2. $\nu_{q}$ is absolutely continuous if and only if $P$ has a nonzero, nonnegative fixed point vanishing outside $I_{q}$.

Proposition 2.2 implies a practical sufficient condition for the absolute continuity. Starting with the characteristic function $f_{0}:=\chi_{I_{q}}$ of the interval $I_{q}$, we introduce a sequence $\left(f_{n}\right) \subset L^{1}(\mathbb{R})$ by the recursive formula

$$
f_{n}:=P f_{n-1}, \quad n=1,2, \ldots,
$$

and we set $f:=\liminf f_{n}$ for brevity.

By Proposition 2.1 all these functions are nonnegative, vanish outside $I_{q}$, and have the same integrals. Applying the Fatou lemma it follows that $\underline{f} \in L^{1}(\mathbb{R})$.

Proposition 2.3. If $\int \underline{f} d x>0$, then $\nu_{q}$ is absolutely continuous.

Proof. We have

$$
\begin{aligned}
& \liminf f_{n}=\liminf \frac{q}{2}\left(f_{n-1}(q x)+f_{n-1}(q x-1)\right) \\
& \geq \frac{q}{2}\left(\liminf f_{n-1}(q x)+\liminf f_{n-1}(q x-1)\right),
\end{aligned}
$$

i.e., $f \geq P f$. Since the integral of $f-P f$ vanishes by Proposition 2.1, we conclude that $P \underline{f}=\underline{f}$. Since $\underline{f} \neq 0$ by assumption, applying the preceding proposition we conclude that $\bar{\nu}_{q}$ is absolutely continuous.

Based on the theory of non-integer base expansions, we will deduce from Proposition 2.3 some more explicit sufficient conditions. Following [7], let us consider the set of numbers $\{p(q)\}$ where $p$ runs over all Newman polynomials, i.e., polynomials with coefficients 0 and 1 , and let us enumerate them into a non-decreasing sequence $\left(y_{n}\right)$. More explicitly, consider the binary expansion $k=\sum a_{i} 2^{i}$ of each nonnegative integer $k$, and set $x_{k}:=\sum a_{i} q^{i}$. Then $x_{k} \rightarrow \infty$, so that the sequence $\left(x_{k}\right)$ may be rearranged into a nondecreasing sequence $\left(y_{k}\right)$.

We have $y_{0}=0, y_{1}=1, y_{2}=q, y_{k} \rightarrow \infty$, and $y_{k+1}-y_{k} \leq 1$ for all $k$. We recall from [8] that if $q=\sqrt{2}$ or if $q \in(1, \sqrt{2})$ is transcendental, then $y_{k+1}-y_{k} \rightarrow 0$. On the other hand, this relation does not hold if $q$ is a Pisot number.

It was proved in [9] that $y_{k+1}-y_{k} \rightarrow 0$ for each $q$ sufficiently close to 1 . This was improved in [1] as follows:

Theorem 2.4. If $1<q \leq 2^{1 / 3}$, then $y_{k+1}-y_{k} \rightarrow 0$. 
See [7], [9], [1], [10] for more results on the validity of this convergence relation.

Learning the results of [9], Kahane indicated in a letter to the second author that this should be related to the problem of absolute continuity of Bernoulli convolutions. He did not elaborate further upon his idea. The following result confirms his intuition on the close relationship between the two problems:

Proposition 2.5. If the sequence $\left(y_{k+1}^{\log _{q} 2}-y_{k}^{\log _{q} 2}\right)$ is bounded, then $\nu_{q}$ is absolutely continuous.

The assumption of the proposition is equivalent to two other useful properties:

LEMMA 2.6. For any fixed real numbers $1<q<2$ and $c>1$ the three sequences of general terms

$$
y_{k}^{c-1}\left(y_{k+1}-y_{k}\right), \quad y_{k+1}^{c-1}\left(y_{k+1}-y_{k}\right) \quad \text { and } \quad y_{k+1}^{c}-y_{k}^{c}
$$

are bounded or unbounded at the same time.

PRoOF. We recall from [7] that $y_{k} \rightarrow \infty$, and $y_{k+1}-y_{k} \leq 1$ for all $k$. Hence $y_{k+1} / y_{k} \rightarrow 1$, and the equiboundedness of the first two sequences follows from the relation

$$
\frac{y_{k+1}^{c-1}\left(y_{k+1}-y_{k}\right)}{y_{k}^{c-1}\left(y_{k+1}-y_{k}\right)}=\left(\frac{y_{k+1}}{y_{k}}\right)^{c-1} \rightarrow 1 \text {. }
$$

Next, applying the Lagrange mean value formula we obtain the inequalities

$$
c y_{k}^{c-1}\left(y_{k+1}-y_{k}\right) \leq y_{k+1}^{c}-y_{k}^{c} \leq c y_{k+1}^{c-1}\left(y_{k+1}-y_{k}\right) .
$$

This shows the equiboundedness of the third sequence.

ExAMPLES. The assumption of Proposition 2.5 is obviously satisfied for $q=2$ because then $y_{k}^{\log _{q} 2}=y_{k}=k$ for all $k$.

One of the equivalent properties of Lemma 2.6 is also satisfied for $q=$ $\sqrt{2}{ }^{1}$ Indeed, by [9, Proposition 3.4] there exists a positive constant $c$ such that if $k$ is sufficiently large and $y_{k}=x-c / x$, then $y_{k+1}-y_{k}<2 c / x$. Then we have

$$
\left(y_{k+1}-y_{k}\right) y_{k}^{-1+\log _{q} 2} \leq \frac{2 c}{x}\left(x-\frac{c}{x}\right) \leq 2 c,
$$

so that the condition is satisfied with $\gamma=2 c$.

\footnotetext{
${ }^{1}$ It was already proven in Kershner-Wintner [15] that $\nu_{q}$ is absolutely continuous for every $q=2^{1 / m}, m=2,3, \ldots$
} 
On the other hand, Proposition 2.5 does not apply for any $q \in\left(\frac{1+\sqrt{5}}{2}, 2\right)$ because then $y_{k+1}-y_{k}=1$ for infinitely many indices $k$ by [7, Theorem 4(b)] and [8, Page 202, property (b)], and

$$
y_{k+1}^{\log _{q} 2}-y_{k}^{\log _{q} 2} \rightarrow \infty
$$

for these indices because $y_{k} \rightarrow \infty$ and $\log _{q} 2>1$.

Proposition 2.5 will be deduced from the following stronger theorem:

TheOREm 2.7. Let $1<q \leq 2$. If there exist two positive constants $\alpha$ and $t_{0}$ such that ${ }^{2}$

$$
\#\left\{k: y_{k} \in[t-1, t]\right\} \geq \alpha t^{\left(\log _{q} 2\right)-1}
$$

for all $t \geq t_{0}$, then $\nu_{q}$ is absolutely continuous.

REMARK. Since $y_{0}=0, y_{k} \rightarrow \infty$, and $y_{k+1}-y_{k} \leq 1$ for all $k=0,1, \ldots$, we have

$$
\#\left\{k: y_{k} \in[t-1, t]\right\} \geq 1
$$

for all $t>0$. Therefore, if the conditions of the proposition are satisfied, then by choosing a suitable smaller $\alpha$ the inequalities (2.1) will hold for all $t>0$.

Proof. We recall from [14] that

$$
f_{n}(x)=\frac{q^{n}}{2^{n}} \sum_{p} \chi_{\left[\frac{p(q)}{q^{n}}, \frac{p(q)}{q^{n}}+\frac{1}{q^{n}(q-1)}\right]}(x), \quad n=0,1 \ldots,
$$

where $p$ runs over the Newman polynomials of degree $<n$. For each fixed $x$, the sum on the right side is equal to the number of Newman polynomials $p$ of degree $<n$ for which

$$
p(q) \leq q^{n} x \leq p(q)+\frac{1}{q-1} .
$$

If $x<1$, then the restriction "of degree $<n$ " may be omitted because $q^{n} x<q^{n}$ and therefore no Newman polynomial of degree $\geq n$ can satisfy the first inequality $p(q) \leq q^{n} x$. Therefore for $x<1$ this sum is equal to the number of indices $k$ for which

$$
y_{k} \leq q^{n} x \leq y_{k}+\frac{1}{q-1},
$$

\footnotetext{
${ }^{2}$ As usual, the symbol \#A denotes the number of elements of the set $A$.
} 
or equivalently

$$
q^{n} x-\frac{1}{q-1} \leq y_{k} \leq q^{n} x .
$$

Fix $\beta \in(0,1)$ arbitrarily, and let $x \in(\beta, 1)$. If $n$ is sufficiently large so that $q^{n} \beta \geq t_{0}$, then we may apply our hypothesis with $t:=q^{n} x$ to conclude that

$$
\begin{gathered}
f_{n}(x)=\frac{q^{n}}{2^{n}} \#\left\{k: y_{k} \in\left[q^{n} x-\frac{1}{q-1}, q^{n} x\right]\right\} \\
\geq \frac{q^{n}}{2^{n}} \#\left\{k: y_{k} \in\left[q^{n} x-1, q^{n} x\right]\right\} \geq \frac{q^{n}}{2^{n}} \alpha\left(q^{n} x\right)^{\left(\log _{q} 2\right)-1}=\alpha x^{\left(\log _{q} 2\right)-1} .
\end{gathered}
$$

Hence

$$
\int \underline{f} d x \geq \int_{\beta}^{1} \alpha x^{\left(\log _{q} 2\right)-1} d x>0 .
$$

If $q$ satisfies the hypotheses of the preceding theorem, then all roots $q^{1 / m}$, $m=2,3, \ldots$ also satisfy them (with different constants $\alpha$ and $t_{0}$ ):

Proposition 2.8. If $q \in(1,2]$ and $\alpha>0$ satisfy the inequalities (2.1), then $p:=q^{1 / m}$ also satisfies the corresponding inequalities with suitable positive constants $\alpha_{m}$ and $t_{0, m}$ for each $m=2,3, \ldots$.

Consequently, the measures $\nu_{q^{1 / m}}$ are also absolutely continuous.

Proof. If we denote by $\left(y_{k}\right)$ and $\left(z_{k}\right)$ the sequences associated with $q$ and $p:=q^{1 / m}$, respectively, then $\left(z_{k}\right)$ is a non-decreasing enumeration of the sums of the form

$$
y_{k_{1}}+y_{k_{2}} p+\cdots+y_{k_{m}} p^{m-1}
$$

where $k_{1}, \ldots, k_{m}$ run independently over the nonnegative integers. Therefore it suffices to find a positive constant $\alpha_{m}$ such that the inequalities

$$
\#\left\{\left(k_{1}, \ldots, k_{m}\right): y_{k_{1}}+y_{k_{2}} p+\cdots+y_{k_{m}} p^{m-1} \in[t-1, t]\right\} \geq \alpha_{m} t^{\left(\log _{p} 2\right)-1}
$$

hold for all sufficiently large real numbers $t$.

Fix a positive real number $\varepsilon$ satisfying $(3 m-2) \varepsilon \leq 1$. Then we have

$$
\begin{aligned}
& \#\left\{\left(k_{1}, \ldots, k_{m}\right): y_{k_{1}}+y_{k_{2}} p+\cdots+y_{k_{m}} p^{m-1} \in[t-1, t]\right\} \\
& \geq \prod_{j=2}^{m} \#\left\{k_{j}: y_{k_{j}} p^{j-1} \in[\varepsilon t, 3 \varepsilon t]\right\} \\
& \quad \times \#\left\{k_{1}: y_{k_{1}} \in\left[t-1-\sum_{j=2}^{m} y_{k_{j}} p^{j-1}, t-\sum_{j=2}^{m} y_{k_{j}} p^{j-1}\right]\right\}
\end{aligned}
$$




$$
\begin{gathered}
=\prod_{j=2}^{m} \#\left\{k_{j}: y_{k_{j}} \in\left[p^{1-j} \varepsilon t, 3 p^{1-j} \varepsilon t\right]\right\} \\
\times \#\left\{k_{1}: y_{k_{1}} \in\left[t-1-\sum_{j=2}^{m} y_{k_{j}} p^{j-1}, t-\sum_{j=2}^{m} y_{k_{j}} p^{j-1}\right]\right\} .
\end{gathered}
$$

If $t \geq t_{0}$ is sufficiently large, then for each $j=2, \ldots, m, p^{1-j} \varepsilon t \geq t_{0}$, each interval $\left[p^{1-j} \varepsilon t, 3 p^{1-j} \varepsilon t\right]$ contains more than $p^{1-j} \varepsilon t$ disjoint unit intervals, and

$$
t-\sum_{j=2}^{m} y_{k_{j}} p^{j-1} \geq t-(m-1) 3 \varepsilon t \geq \varepsilon t \geq t_{0}
$$

so that

$$
\begin{gathered}
\#\left\{\left(k_{1}, \ldots, k_{m}\right): y_{k_{1}}+y_{k_{2}} p+\cdots+y_{k_{m}} p^{m-1} \in[t-1, t]\right\} \\
\geq\left(\prod_{j=2}^{m}\left(p^{1-j} \varepsilon t\right) \alpha\left(p^{1-j} \varepsilon t\right)^{\left(\log _{q} 2\right)-1}\right) \alpha(\varepsilon t)^{\left(\log _{q} 2\right)-1} \\
=\alpha^{m}\left(\prod_{j=2}^{m} p^{(1-j) \log _{q} 2}\right)(\varepsilon t)^{m\left(\log _{q} 2\right)-1} \\
=\alpha^{m}\left(\prod_{j=2}^{m} p^{(1-j) \log _{q} 2}\right)(\varepsilon t)^{\left(\log _{p} 2\right)-1}=\alpha_{m} t^{\left(\log _{p} 2\right)-1}
\end{gathered}
$$

with

$$
\alpha_{m}:=\alpha^{m}\left(\prod_{j=2}^{m} p^{(1-j) \log _{q} 2}\right) \varepsilon^{\left(\log _{p} 2\right)-1}>0 .
$$

Finally we show that the hypotheses of Proposition 2.5 imply those of Theorem 2.7:

LEMMA 2.9. If the sequence $\left(y_{k+1}^{\log _{q} 2}-y_{k} \log _{q} 2\right)$ is bounded, then there exist two positive constants $\alpha$ and $t_{0}$ such that

$$
\#\left\{k: y_{k} \in[t-1, t]\right\} \geq \alpha t^{\left(\log _{q} 2\right)-1}
$$

for all $t \geq t_{0}$.

Proof. If $q=2$, then (2.3) is satisfied with $\alpha=1$ for all $t>0$. Henceforth we assume that $1<q<2$. 
By Lemma 2.6 there exists a positive constant $c_{1}$ such that

for all $k \geq 1$. Hence

$$
y_{k}-y_{k-1} \leq \frac{c_{1}}{y_{k}^{\left(\log _{q} 2\right)-1}}
$$

$$
y_{k}-y_{k-1} \leq \frac{c_{1}}{(t-1)^{\left(\log _{q} 2\right)-1}}=: \varepsilon(t)
$$

whenever $k \geq 1$ and $y_{k} \geq t-1$.

It follows that

$$
\#\left\{k: y_{k} \in[t-1, t]\right\} \geq \frac{1-\varepsilon(t)}{\varepsilon(t)}=\frac{(t-1)^{\left(\log _{q} 2\right)-1}}{c_{1}}-1 .
$$

If $t$ is sufficiently large, say $t \geq t_{0}$, then

$$
\frac{(t-1)^{\left(\log _{q} 2\right)-1}}{c_{1}}-1 \geq \frac{(t / 2)^{\left(\log _{q} 2\right)-1}}{c_{1}}
$$

because $\left(\log _{q} 2\right)-1>0$, so that $(2.3)$ is satisfied with

$$
\alpha=\frac{1}{2^{\left(\log _{q} 2\right)-1} c_{1}} .
$$

We end this paper with some open problems.

(1) Is the assumption $R>\sqrt{\mu}$ in Theorem 1.2 optimal?

(2) Find more examples of $q \in(1,2)$ for which the sequence

is bounded.

$$
\left(y_{k+1}^{\log _{q} 2}-y_{k}^{\log _{q} 2}\right)
$$

(3) More generally, investigate the set

$$
\left\{(q, c) \in(1,2] \times[1, \infty) \text { : the sequence }\left(y_{k+1}^{c}-y_{k}^{c}\right) \text { is bounded }\right\} .
$$

Acknowledgements. We are grateful to the referee and to Sz. Révész for their useful suggestions and corrections to the first version of this paper.

\section{References}

[1] S. Akiyama and V. Komornik, Discrete spectra and Pisot numbers, J. Number Theory, 133 (2013), 375-390.

[2] C. Baiocchi, V. Komornik and P. Loreti, Théorèmes du type Ingham et application à la théorie du contrôle, C. R. Acad. Sci. Paris Sér. I Math., 326 (1998), 453-458. 
[3] C. Baiocchi, V. Komornik and P. Loreti, Ingham type theorems and applications to control theory, Bol. Un. Mat. Ital. B, (8), 2 (1999), 33-63.

[4] K. Dajani and M. de Vries, Measures of maximal entropy for random $\beta$-expansions, J. Eur. Math. Soc., 7 (2005), 51-68.

[5] P. Erdős, On a family of symmetric Bernoulli convolutions, Amer. J. Math., 61 (1939), 974-976.

[6] P. Erdős, On the smoothness properties of a family of Bernoulli convolutions, Amer. J. Math., 62 (1940), 180-186.

[7] P. Erdős, I. Joó and V. Komornik, Characterization of the unique expansions $1=$ $\sum q^{-n_{i}}$ and related problems, Bull. Soc. Math. France, 118 (1990), 377-390.

[8] P. Erdős, I. Joó and V. Komornik, On the sequence of numbers of the form $\varepsilon_{0}+\varepsilon_{1} q+$ $\cdots+\varepsilon_{n} q^{n}, \varepsilon_{i} \in\{0,1\}$, Acta Arith., 83 (1998), 201-210.

[9] P. Erdös and V. Komornik, Developments in noninteger bases, Acta Math. Hungar., 79 (1998), 57-83.

[10] De-Jun Feng, On the topology of polynomials with bounded integer coefficients, J. Eur. Math. Soc., 18 (2016), 181-193.

[11] B. Jessen and A. Wintner, Distribution functions and the Riemann zeta function, Trans. Amer. Math. Soc., 38 (1935), 48-88.

[12] J.-P. Kahane, Pseudo-périodicité et séries de Fourier lacunaires, Ann. Sci. École Norm. Sup., 79 (1962), 93-150.

[13] J.-P. Kahane, Sur la distribution de certaines séries aléatoires, in: Colloque de Théorie des Nombres (Univ. Bordeaux, Bordeaux, 1969), Bull. Soc. Math. France, Mém. No. 25, Soc. Math. France (Paris, 1971), 119-122.

[14] T. Kempton, Counting $\beta$-expansions and the absolute continuity of Bernoulli convolutions, Monatsh. Math., 171 (2013), 189-203.

[15] R. Kershner and A. Wintner, On symmetric Bernoulli convolutions, Amer. J. Math., 57 (1935), 541-548.

[16] V. Komornik, Expansions in noninteger bases, Tutorial and review, Workshop on Numeration, Lorentz Center, Leiden, June 7-11, 2010, Integers, 11B (2011), A9, 1-30.

[17] V. Komornik and P. Loreti, Fourier Series in Control Theory, Springer (New York, 2005).

[18] B. Solomyak, On the random series $\sum \pm \lambda^{n}$ (an Erdős problem), Ann. of Math. (2), 142 (1995), 611-625. 